\title{
Quantum Correlations of Spin-1 Atoms in an Optical Lattice ${ }^{1}$
}

\author{
B. Öztop ${ }^{a}$, M. Ö. Oktel ${ }^{a}$, and Ö. E. Müstecaplığlu \\ ${ }^{a}$ Department of Physics, Bilkent University, 06800 Bilkent, Ankara, Turkey \\ ${ }^{b}$ Department of Physics, Koç University, 34450 Sartyer, Istanbul, Turkey \\ e-mail: boztop@fen.bilkent.edu.tr \\ Received September 30, 2008
}

\begin{abstract}
In this work, we investigate the system of cold spin-1 atoms in a one dimensional optical lattice in relation with squeezing and entanglement. By using the corresponding Bose-Hubbard Hamiltonian, both superfluid and Mott-insulator phases are studied by using numerical methods in the mean-field approximation. To observe the presence of entanglement, we used a squeezing measure as a criterion for quantum correlations. We further investigate the two interaction regimes, namely ferromagnetic and antiferromagnetic in the case of zero and nonzero but very small angle between the counterpropagating laser beams that form the optical lattice. States in the superfluid phase are calculated analytically by using the perturbation theory.
\end{abstract}

PACS numbers: 03.75.Lm, 03.75.Mn, 03.67.Bg

DOI: $10.1134 / \mathrm{S} 1054660 \mathrm{X} 0904015 \mathrm{X}$

\section{INTRODUCTION}

It was shown by using the Bose-Hubbard model that bosons with short-ranged repulsive interactions in a periodic potential can undergo a quantum phase transition between the superfluid and Mott-insulator phases, that exists at sufficiently low temperatures [1]. Later on Bose-Hubbard model was used for a system of cold bosonic atoms in an optical lattice [2]. It was experimentally realized [3] and theoretically examined further [4]. Systems of multi-component Bose-Einstein condensates (BECs) in optical lattices [5] has been investigated as well as some topics such as quantum phase transitions of spin-2 bosons [6], two-component condensates [7], and spin-1 bosons with coupled ground states [8].

Spin squeezing is known as being present for those states with fluctuations below the standard quantum limit in one of the spin components. In [9], it has been shown that the spin squeezed states of a spin-s system correspond to the correlated states of a system that consists of $2 s$ spin- $1 / 2$ substructures. This result shows the relationship between the concept of spin squeezing and entanglement, the concept that is strongly related to the existence of quantum correlations. Spin squeezing has been shown to be possible in some many-particle systems with various interactions like for BECs in an external trap [10], in many atomic gas models [11, 12] and for atoms in optical lattices [13].

The main objective of this work is to investigate the possibility and conditions of squeezing in the pseudospin for a system of spin-1 bosons with coupled ground states in an optical lattice, that is the system studied in

\footnotetext{
${ }^{1}$ The article is published in the original.
}

$[8,14]$. The paper is organized as follows. We first we explain the system under consideration and give the Bose-Hubbard Hamiltonian in the mean-field approximation in Section 2. Later on, the numerical diagonalization of this Hamiltonian and the measure of squeezing that we use are mentioned in Section 3. Then in Section 4 the results for different interaction regimes are given. Finally in Section 5 we use perturbation theory to find some superfluid states analytically starting from mean-field Mott-insulator states and conclude in Section 6.

\section{MODEL}

In the present paper, we are interested in a system of neutral spin-1 bosonic atoms of fixed mass. Due to their polarizability, hyperfine states of the atoms $(F=1, m=$ $0, \pm 1$ ) become the degenerate ground and excited states when the transitions between them are induced by some laser field as they are placed in a one-dimensional optical lattice. $F=1$ hyperfine states of the atoms are coupled via $V$ and $\Lambda$-type transitions due to the present laser field, and the excited state is adiabatically eliminated by imposing large detuning. It is assumed that the atoms remain in the lowest Bloch bands, which is due to the relatively large energy difference between the lowest and first excited bands. In this approximation, expansion of the atomic spinor-field operator in the Wannier basis is legitimate [8]. So, the Hamiltonian of the system can be written in terms of a sum over two lowest energy eigenmodes, 0 and $\Lambda$.

The resulting Bose-Hubbard Hamiltonian for spin1 bosons with coupled ground states in a one-dimen- 
sional optical lattice is given by

$$
\begin{gathered}
\hat{H}_{B H}=-\sum_{\sigma=0, \Lambda}\left|J_{\sigma}\right| \sum_{\langle i, j\rangle} \hat{a}_{\sigma i}^{\dagger} \hat{a}_{\sigma j} \\
+\sum_{\sigma=0, \Lambda} \frac{U_{\sigma}}{2} \sum_{i} \hat{n}_{\sigma i}\left(\hat{n}_{\sigma i}-1\right)+K \sum_{i} \hat{n}_{0 i} \hat{n}_{\Lambda i} \\
-\frac{|P|}{2} \sum_{i}\left(\hat{a}_{0 i}^{\dagger} \hat{a}_{0 i}^{\dagger} \hat{a}_{\Lambda i} \hat{a}_{\Lambda i}+\hat{a}_{\Lambda i}^{\dagger} \hat{a}_{\Lambda i}^{\dagger} \hat{a}_{0 i} \hat{a}_{0 i}\right) \\
-\delta \sum_{i} \hat{n}_{0 i}-\mu \sum_{\sigma=0, \Lambda} \sum_{i} \hat{n}_{\sigma i},
\end{gathered}
$$

where $J_{\sigma}$ are the tunneling parameters, $U_{\sigma}, K$, and $P$ are the atomic interaction parameters due to repulsive interaction of the condensate atoms and spin-changing collisions. $\delta$ is a parameter that shows the difference between the mean energies of the eigenmodes 0 and $\Lambda$. The parameters in the Hamiltonian (1) can be given in terms of Wannier spinors [8]. As a result, all these parameters depend on the angle $\theta$, that is the angle between the polarization vectors of the two counterpropagating linearly polarized laser beams, which create the optical lattice, $\mu$ is the chemical potential, $\hat{a}_{\sigma i}^{\dagger}$ and $\hat{a}_{\sigma i}$ are atom annihilation and creation operators of type $\sigma$ at lattice site $i$ and $\hat{n}_{\sigma i}=\hat{a}_{\sigma i}^{\dagger} \hat{a}_{\sigma i}$.

In the mean-field approximation [15]

$$
\hat{a}_{\sigma i}^{\dagger} \hat{a}_{\sigma j} \approx \psi_{\sigma}\left(\hat{a}_{\sigma j}+\hat{a}_{\sigma i}^{\dagger}\right)-\psi_{\sigma}^{2}
$$

the single site Hamiltonian becomes

$$
\begin{gathered}
\hat{H}_{B H}^{\mathrm{MF}}=-2 \sum_{\sigma=0, \Lambda} J_{\sigma}\left[\left(\hat{a}_{\sigma}+\hat{a}_{\sigma}^{\dagger}\right) \psi_{\sigma}-\psi_{\sigma}^{2}\right] \\
+\sum_{\sigma=0, \Lambda} \frac{U_{\sigma}}{2} \hat{n}_{\sigma}\left(\hat{n}_{\sigma}-1\right)+K \hat{n}_{0} \hat{n}_{\Lambda} \\
-\frac{|P|}{2}\left(\hat{a}_{0}^{\dagger} \hat{a}_{0}^{\dagger} \hat{a}_{\Lambda} \hat{a}_{\Lambda}+\hat{a}_{\Lambda}^{\dagger} \hat{a}_{\Lambda}^{\dagger} \hat{a}_{0} \hat{a}_{0}\right)-\delta \hat{n}_{0}-\mu \sum_{\sigma=0, \Lambda} \hat{n}_{\sigma},
\end{gathered}
$$

with $\psi_{\sigma}=\left\langle\hat{a}_{\sigma j}\right\rangle$ assumed real [8].

For this system at hand, we can introduce the generators of su(2) isospin algebra

$$
\begin{aligned}
& \hat{T}_{1}=\frac{1}{2}\left(\hat{a}_{\Lambda}^{\dagger} \hat{a}_{0}+\hat{a}_{0}^{\dagger} \hat{a}_{\Lambda}\right), \\
& \hat{T}_{2}=\frac{i}{2}\left(\hat{a}_{\Lambda}^{\dagger} \hat{a}_{0}-\hat{a}_{0}^{\dagger} \hat{a}_{\Lambda}\right), \\
& \hat{T}_{3}=\frac{1}{2}\left(\hat{a}_{0}^{\dagger} \hat{a}_{0}-\hat{a}_{\Lambda}^{\dagger} \hat{a}_{\Lambda}\right),
\end{aligned}
$$

in terms of which the mean-field Hamiltonian (3) can be expressed as

$$
\begin{gathered}
\hat{H}_{B H}^{\mathrm{MF}}=-2 \sum_{\sigma=0, \Lambda} J_{\sigma}\left[\left(\hat{a}_{\sigma}+\hat{a}_{\sigma}^{\dagger}\right) \psi_{\sigma}-\psi_{\sigma}^{2}\right] \\
+\frac{U_{\Sigma}}{2} \hat{T}_{3}^{2}+(K-|P|) \hat{T}_{1}^{2}+(K+|P|) \hat{T}_{2}^{2}+\frac{U_{\Sigma}}{8} \hat{n}^{2} \\
-\left(\frac{K}{2}+\mu+\frac{U_{\Sigma}}{4}+\frac{\delta}{2}\right) \hat{n}-\left(\frac{\Delta U}{2}+\delta\right) \hat{T}_{3}+\frac{\Delta U}{2} \hat{n} \hat{T}_{3},
\end{gathered}
$$

where $\Delta U=U_{0}-U_{\Lambda}, U_{\Sigma}=U_{0}+U_{\Lambda}$, and $\hat{n}=\hat{n}_{0}+\hat{n}_{\Lambda}$. Spin dependent interaction terms in this Hamiltonian are very similar to that of the generalized LipkinMeshkov-Glick (LMG) model [16, 17], or its special case of the two-axis twisting model [9]. It was shown that these models can generate spin squeezing [9] and multiparticle entanglement $[11,16]$. The tunnelling and collision effects are also contained in the Hamiltonian (5) as well as the generalized LMG interaction terms.

For the lattice parameter $\theta=0$, the two modes have the same energy and the interaction and tunnelling parameters are same for the two modes, and $\delta=0$ [8]. By using these relations, the Hamiltonian can be simplified further

$$
\begin{aligned}
\hat{\mathscr{H}}_{\mathrm{af}(\mathrm{f})} & =-2 J \sum_{\sigma=0, \Lambda}\left[\left(\hat{a}_{\sigma}^{\dagger}+\hat{a}_{\sigma}\right) \psi_{\sigma}-\psi_{\sigma}^{2}\right] \\
& +2\left(U \hat{T}^{2}+P \hat{T}_{2(1)}^{2}\right)+\alpha \hat{n},
\end{aligned}
$$

for both antiferromagnetic $(P>0)$ and ferromagnetic $(P<0)$ interactions [14], where $\hat{T}^{2}=\hat{n}^{2} / 4+\hat{n} / 2$ with $\alpha=-3 U / 2-P / 2-\mu$. In this case, the interaction becomes a single-axis twisting type which was also mentioned in [9]. In the general case of the model system above, particle entanglement exists for atoms in the non-degenerate ground state modes, which become degenerate for the case of $\theta=0$.

\section{METHODS}

In our work, to identify entanglement (or squeezing) we use a criterion that was introduced originally for an atomic Bose-Einstein condensate (BEC) [10]. If the squeezing parameter

$$
\xi_{\alpha}^{2}=\frac{N\left(\Delta J_{\alpha}\right)^{2}}{\left\langle J_{\beta}\right\rangle^{2}+\left\langle J_{\gamma}\right\rangle^{2}}
$$

is smaller than 1, the two mode bosonic many atom state under consideration is spin squeezed along the direction of $\alpha$. $\mathbf{J}$ is the total isospin operator, $\alpha, \beta$, and $\gamma$ denote three orthogonal axes. We examine spin squeezing for the on-site isospin algebra by calculating the variance and expectation values of the corresponding generators $T_{i}$ in (4). This would show the existence 